\title{
Nontraumatic Splenic Rupture due to Infectious Mononucleosis
}

\author{
Elliot A. Frank a,*, James R LaFleur b,c, Stanley Okosun d \\ a Lincoln Family Medicine Residency Program, Lincoln Medical Education Partnership, Lincoln, NE, USA \\ ${ }^{b}$ Idaho State University, Department of Physician Assistant Studies, Idaho State University, Idaho, ID, USA \\ ${ }^{c}$ The College of Idaho, Department of Physician Assistant Studies, Idaho, ID, USA \\ d Bryan Trauma/Acute Care surgery, Lincoln, Nebraska, NE, USA
}

\section{Article history:}

Received: March 15, 2018

Revised: August 3, 2018

Accepted: August 5, 2018

\author{
${ }^{*}$ Corresponding Author: \\ Elliot A. Frank \\ Lincoln Family Medicine \\ Residency, Lincoln, NE, USA \\ E-mail: elliotfrankmd@gmail.com \\ ORCID \\ https://orcid.org/0000-0003-3424-9093
}

\begin{abstract}
A 19-year-old otherwise healthy male presented to the Emergency Department with left upper quadrant abdominal pain having felt a "pop" in his abdomen which was followed by nausea and lightheadedness. There was no evidence of trauma but 3 weeks earlier he began with symptoms of a sore throat and nasal congestion without cough. On subsequent investigation, given the patient's acute abdominal pain, abnormal vitals and a non-diagnostic computed tomography scan, an emergent exploratory laparotomy was performed. There was $600 \mathrm{~mL}$ of blood evacuated from the abdomen. A 643-gram inflamed and ruptured spleen was identified and removed, and follow-up lab work was positive for heterophile antibody. This report describes spontaneous splenic rupture caused by infectious mononucleosis and compares characteristics of traumatic versus non-traumatic cases.
\end{abstract}

Keywords: Epstein-Barr virus, infectious mononucleosis, splenectomy, splenic rupture

\section{Introduction}

Infectious mononucleosis is a common viral infection primarily caused by the Epstein-Barr virus (EBV). Symptoms typically begin from 4-6 weeks after EBV infection and include symptoms of a sore throat with swollen lymph nodes, extreme fatigue, fevers, head and body aches, hepatomegaly, splenomegaly and rash [1]. Infection occurs primarily through body fluids, sexual contact and fomites. In developing nations, $>90 \%$ of children have already been infected by EBV by 5-9 years of life, versus $50 \%$ of similar-aged children in developed nations [2]. In the United States, $82.9 \%$ of teens 18 -19 years old tested seropositive for EBV [3], and only 25\% of those infections develop into infectious mononucleosis [1]. Occurring in less than $0.5 \%$ of cases of infectious mononucleosis, splenic rupture is a rare complication [4]. The purpose of this study was to show that the course of a common illness can have important surgical consequences, and clinicians need to be alert to the presence of infectious mononucleosis, even in non-traumatic situations.

\section{Case Report}

A 19-year-old otherwise healthy male presented to clinic with a 1-week history of a sore throat and nasal congestion without a cough. He was prescribed a course of Cefuroxime for acute rhinosinusitis but there was no improvement in his condition. Two weeks later, he was playing video games when he felt a mild "pop" in his abdomen followed by nausea and lightheadedness. He was evaluated in a local Emergency Department and sent home with amoxicillin after a positive 
rapid strep test. At home, his nausea and lightheadedness worsened and he developed left upper quadrant abdominal pain.

He presented at a second hospital, where his vital signs were measured. His pulse was 118 beats/min, respiration 21 breaths/ min, blood pressure $107 / 64 \mathrm{mmHg}$, and his temperature was $36.6^{\circ} \mathrm{C}$. A physical examination showed a well-developed, pale and diaphoretic teenager in moderate distress, lying in a right lateral decubitus position. He was unable to lay in a supine position or change position. His abdomen was normal on inspection, with normal bowel sounds, and it was rigid with acute tenderness and guarding in all quadrants. There was no ecchymosis on his back. There was mild cervical lymphadenopathy and exudative tonsillar hypertrophy. Laboratory investigation revealed leukocytosis (8.7 109/ L), normocytic anemia (hemoglobin $6.95 \mathrm{mMol} / \mathrm{L}$ ) and mild hyperbilirubinemia (total bilirubin $41.04 \mu \mathrm{Mol} / \mathrm{L}$ ) with normal transaminases. A computed tomography (CT) scan showed hepatosplenomegaly with a moderate amount of free fluid in the abdomen and pelvis, without the appendix being visualized (Figures 1 and 2).

Given the patient's acute abdomen, abnormal vitals, and non-diagnostic CT scan, an emergency exploratory laparotomy was performed. There was $600 \mathrm{~mL}$ of blood evacuated from his abdomen. An inflamed and ruptured spleen (643 g) was identified and removed (Figure 3), and follow-up lab work was positive for heterophile antibody. The post-operative recovery was unremarkable.

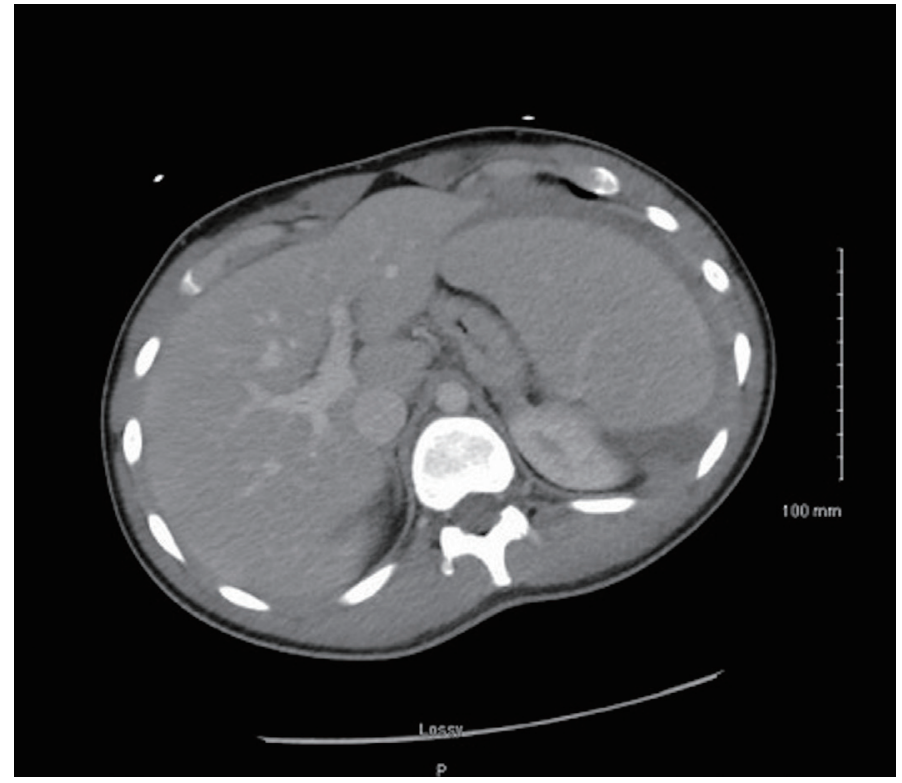

Figure 1. Transverse section of computed tomography with contrast showing hepatomegaly and splenomegaly with surrounding free fluid vs edema.

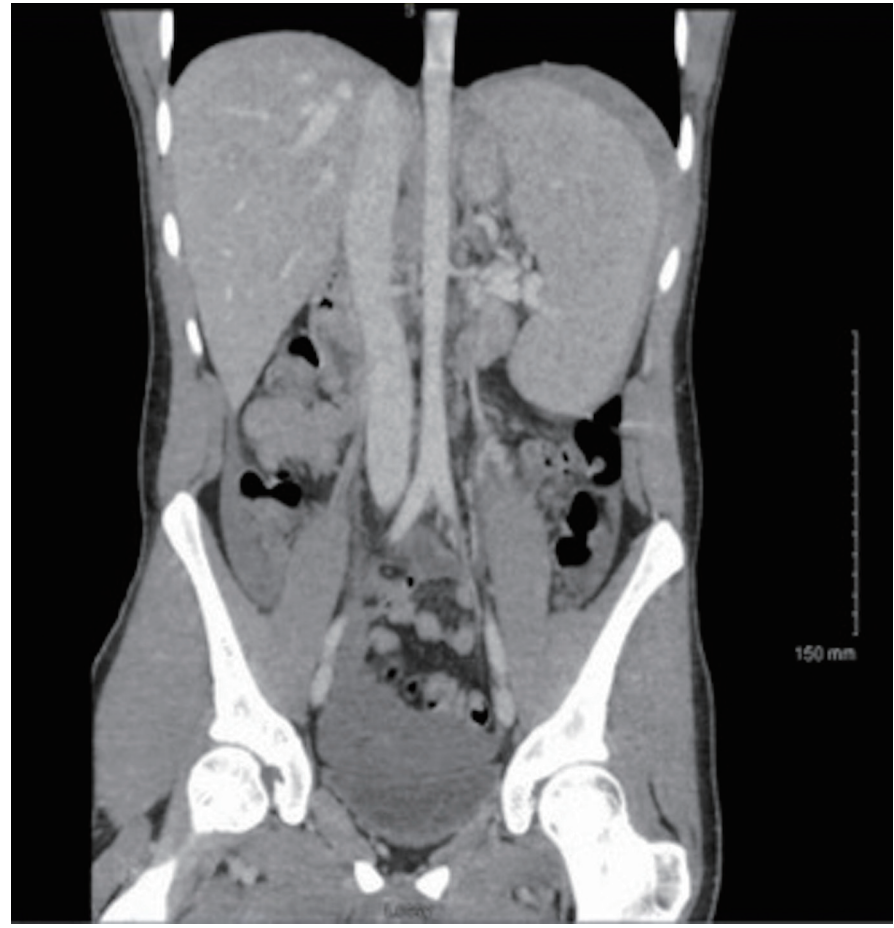

Figure 2. Coronal section of computed tomography with contrast showing hepatomegaly and splenomegaly with surrounding free fluid vs edema.

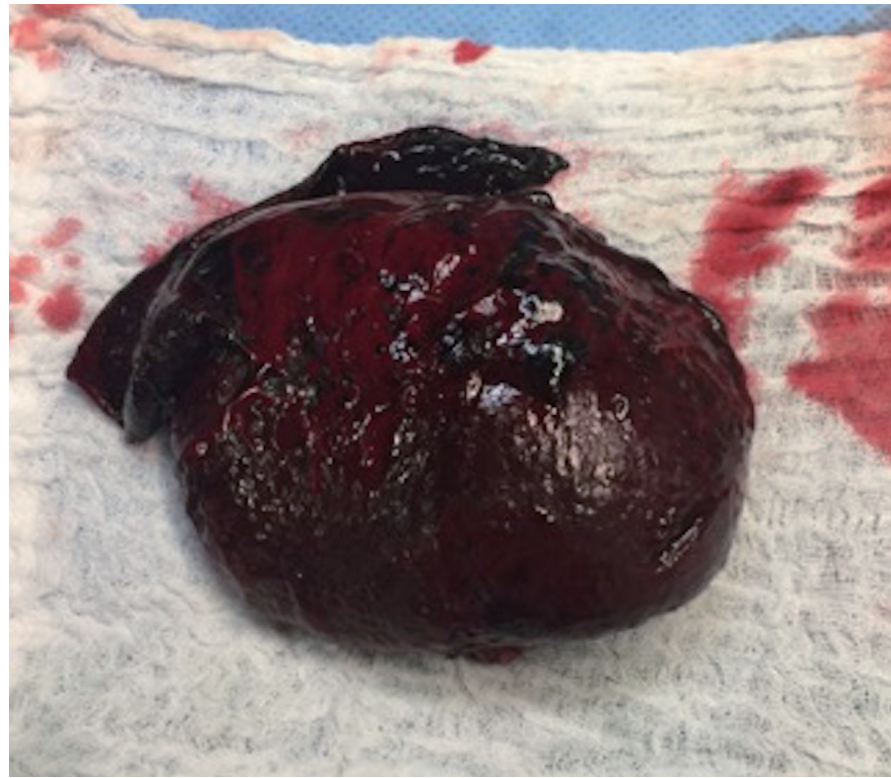

Figure 3. Ruptured spleen after splenectomy, measuring 643 grams. 


\section{Discussion}

Unlike in traumatic splenic rupture, the mechanism of rupture in spontaneous cases is unknown. In a series of 613 patients, Aubrey-Bassler and Sowers reported that the most common categories in non-traumatic splenic rupture included infections (malaria, mononucleosis), followed by procedureinduced ruptures (colonoscopy), hematologic (lymphoma and leukemia) and cancers [5]. One study tested patients with confirmed mononucleosis and reported that they all had splenomegaly, suggesting this was a causal factor [6]. Histologically, splenomegaly is due to $\mathrm{T}$ and $\mathrm{B}$ cell infiltration and expansion of the red pulp of the spleen [7], which appears like a lymphoma. There are cases reported where vomiting, coughing, and even sneezing have been identified as triggers for the rupture of the spleen.

The use of a CT scan has allowed the emergence of nonoperative management (waiting and angioembolization) of splenic injury in hemodynamically stable patients, when splenic pathology has been detected [8]. Using the American Association for the Surgery of Trauma (AAST) rating scale, grades of splenic injury correlate well with clinical outcome. Grade I is the least severe (a subcapsular hematoma occupying $<10 \%$ of surface area) and Grade V is the most severe (a shattered and/or devascularized spleen). As grades increase, the success of non-operative management decreases. In 1 study, Grade III injured spleens were associated with $19.6 \%$ of non-operative failure versus a $75 \%$ failure rate in Grade $\mathrm{V}$ injured spleens. A successful non-operative approach was also associated with higher blood pressure, higher hematocrit and a lower quantity of hemoperitoneum. In this current case study, there was significant diagnostic and radiographic uncertainty preventing the use of the AAST rating scale. While the AAST rating scale is not well defined in cases of spontaneous splenic rupture (SSR), data shows that contrast-enhanced ultrasound provides an equivalent grading scale regardless of cause [9]. This same study also reported that, compared with traumatic splenic rupture (TSR), those with SSR were on average 62 versus 44 years old, and had a 4 -week mortality of $36 \%$ vs $0 \%$ [9].

For acute care surgeons, it is important to be aware of organ ruptures in non-traumatic settings. To that end, 1 case series reported 85 cases of mononucleosis requiring splenectomy from 1984 to 2014, where only $14 \%$ of patients had a prior history of trauma leading to splenic rupture [10]. They observed that $70 \%$ were male with an average age of 22 . Abdominal pain was the presenting complaint in $88 \%$ of cases and $67 \%$ required a splenectomy. The mortality rate was $9 \%$ for SSR [10]. In comparison, the mortality rate from TSR was reported retrospectively in 15,388 cases [11]. Patients with isolated splenic injury of AAST Categories I and II, III, IV, and V had $6.9 \%, 7.1 \%, 9.4 \%$, and $22.7 \%$, mortality rate, respectively, with an average of $8.3 \%$ across all groups [11]. The mortality risk of TSR appears similar to that of rupture due to mononucleosis, whereas mortality from other causes of SSR may be far greater [9].

\section{Conflicts of Interest}

No conflicts of interest are disclosed for any of the authors of this report.

\section{References}

[1] Epstein-barr | Mononucleosis | About Mono | CDC [Internet]. [cited 2017 Oct 20]. Available from: https://www.cdc.gov/epstein-barr/about-mono. html.

[2] Takeuchi K, Tanaka-Taya K, Kazuyama Y, Ito YM, Hashimoto S, Fukayama $\mathrm{M}$, et al. Prevalence of Epstein-Barr virus in Japan: Trends and future prediction. Pathol Int 2006;56(3):112 -6.

[3] Dowd JB, Palermo T, Brite J, McDade TW, Aiello A. Seroprevalence of Epstein-Barr Virus Infection in U.S. Children Ages 6-19, 2003-2010. PLOS ONE 2013;8(5):e64921.

[4] Jenson HB. Acute complications of Epstein-Barr virus infectious mononucleosis. Curr Opin Pediatr 2000 Jun;12(3):263-268.

[5] Aubrey-Bassler FK, Sowers N. 613 cases of splenic rupture without risk factors or previously diagnosed disease: a systematic review. BMC Emerg Med 2012;12:11.

[6] Dommerby H, Stangerup SE, Stangerup M, Hancke S. Hepatosplenomegaly in infectious mononucleosis, assessed by ultrasonic scanning. J Laryngol Otol 1986;100(5):573-9.

[7] Siliézar MM, Muñoz CC, Solano-Iturri JD, Ortega-Comunian L, Mollejo M, Montes-Moreno S, et al. Spontaneously Ruptured Spleen Samples in Patients with Infectious Mononucleosis Analysis of Histology and Lymphoid Subpopulations. Am J Clin Pathol 2018;150(4):310-7.

[8] El-Matbouly M, Jabbour G, El-Menyar A, Peralta R, Abdelrahman H, Zarour A, et al. Blunt splenic trauma: Assessment, management and outcomes. Surgeon 2016;14(1):52-8.

[9] Rosling M, Trenker C, Neesse A, Görg C. Spontaneous and Traumatic Splenic Rupture: Retrospective Clinical, B-Mode and CEUS Analysis in 62 Patients. Ultrasound Int Open 2018;4(1):E30 -4.

[10] Bartlett A, Williams R, Hilton M. Splenic rupture in infectious mononucleosis: A systematic review of published case reports. Injury 2016;47(3):531-8.

[11] Tinkoff G, Esposito TJ, Reed J, Kilgo P, Fildes J, Pasquale M, et al. American Association for the Surgery of Trauma Organ Injury Scale I: Spleen, liver, and kidney, validation based on the National Trauma Data Bank. J Am Coll Surg 2008;207(5):646-55. 